\title{
INOVAÇÃO NO DESIGN CONTEMPORÂNEO: APROXIMAÇÕES CONCEITUAIS ENTRE A ENGENHARIA KANSEI E A INTERNET DAS COISAS
}

Erika Yamamoto Lee (erikaylee@gmail.com) - Departamento de Design, Universidade Federal do Paraná.

Virgínia Borges Kistmann (vkistmann@ufpr.br) - Departamento de Design, Universidade Federal do Paraná.

Viviane G. R. El Marghani (viviane.gasparibas@gmail.com) - Departamento de Design, Universidade Federal do Paraná.

\section{RESUMO}

O presente artigo objetiva apresentar uma discussão teórico-analítica a respeito do conceito de inovação no design voltado para a Internet das Coisas e Engenharia Kansei, sob a perspectiva da Inovação no design contemporâneo. As inovações ocorrem quando um novo processo, produto ou serviço é incorporado ao mercado, proporcionando uma vantagem competitiva para a instituição que as administra. A inovação é um dos objetivos da Engenharia Kansei, mas ainda pouco explorada no que se refere à Internet das Coisas. Este trabalho, portanto, busca relacionar o conceito de inovação em design voltado para a Internet das Coisas e para a Engenharia Kansei. Estima-se que a Internet das Coisas irá produzir um trilhão e novecentos mil dólares em produtos e serviços, até o ano de 2020. O trabalho parte do pressuposto de que esses dois objetos de pesquisa podem contribuir na inovação do design. Com essa finalidade, trata os temas a partir da revisão de literatura, obtendo como resultado possiveis relações por meio da definição tanto da Internet das Coisas de modo amplo e foca a Engenharia Kansei Colaborativa no âmbito da geração de inovação no Design atual.

Palavras-chave: Design Colaborativo; Inovação em Design; Engenharia Kansei Colaborativa; Internet das Coisas.

Área: Design e o Desenvolvimento de Produtos.

\section{INTRODUÇÃO}

Este artigo consiste em um ensaio de base teórico-analítica sobre o uso da Engenharia Kansei (KE, sigla utilizada nas pesquisas internacionais: Kansei Engineering) e da Internet das Coisas (IoT, da sigla em inglês) como fontes de inovação associadas ao design. A abordagem ensaística foi selecionada, pois se trata de um tema que traz no seu contexto uma breve abordagem epistemológica contemporânea, não considerada usualmente, no campo da engenharia e do design.

Propõe-se, assim, uma contribuição sobre a complexidade contemporânea, na aproximação de elementos, camadas e estruturas, cujas inter-relações condicionam e redefinem a inovação no design contemporâneo. O objetivo do breve trabalho é levantar uma reflexão sobre a geração de inovação no design atual, salientando os elementos da crescente pesquisa, tanto acadêmica quanto industrial, que investigam e aplicam as novas tecnologias em colaboração. 
A KE tem como objetivo propor novos produtos que inovem, com base na introdução de elementos formais, tecnológicos e conceituais definidos a partir de parâmetros ligados à sistematização das emoções (NAGAMACHI, 2011; SHÜTTE, 2002).

A IoT é um conceito ativo, por meio do qual os usuários se apropriam do território virtual para comunicar, desenvolver produtos ou serviços, na área do design, que gera inovações tangíveis e intangíveis, no capitalismo contemporâneo (DU et al., 2013; KAMIENSKI et al., 2017).

O campo do design vem trazendo a discussão quanto ao design e à emoção, de forma sistematizada, desde Jordan (2000) e Norman (2004), sendo que, nos últimos anos, essas abordagens foram incorporadas por uma série de estudos, com diversas denominações: user experience e o design emocional (nível de processamento de informação), por exemplo, linha de pesquisa que provém da ergonomia, no contexto da experiência do usuário e da usabilidade sensorial com o produto, ou seja, ocupa-se da natureza das emoções nos produtos. No entanto, algumas questões não são abordadas nesses estudos, tais como o pensamento contemporâneo, no capitalismo cognitivo, que faz do designer um pesquisador e gestor, na produção de inovação para um mundo complexo, o qual demanda outros tipos de produtos não exclusivamente materiais.

A breve análise comparativa entre as teorias conceituais da KE e a da IoT permite pensar a atividade do designer contemporâneo, contribuindo no gerenciamento do processo e na produção de inovação, no novo ambiente cotidiano, bem como indagar quanto a possíveis novos estudos relacionados ao uso desses instrumentos tecnológicos associados ao design (design será empregado como o campo ou o desenvolvimento de produtos).

Assim, este artigo almeja, na primeira parte, buscar aproximações conceituais da inovação no design contemporâneo: trata da apropriação dos elementos contemporâneos, como a produção em rede colaborativa no uso da interoperatividade da IoT. Na segunda parte, apresenta breve contextualização dos conceitos da IoT e dos componentes envolvidos. Na terceira parte, desenvolve o conceito da KE e os tipos aplicados para o desenvolvimento da inovação. Atribui-se ênfase ao sexto tipo de KE, o qual se aplica à colaboração, que pode se dar entre homem e máquina, visto que essa máquina pode gerar inovação. Na quarta parte, propõe-se expor um quadro comparativo, o qual implica o desenvolvimento de produto nestes dois conceitos de pesquisa.

Dentre esses dois estudos geradores de inovação no design vigente, formula-se a questão: "É possível construir inovação em rede, considerando a IoT e a KE colaborativa, desenvolvidas por meio da interoperabilidade e da emoção?"

\section{DESENVOLVIMENTO}

A pesquisa em design e a respectiva produção de inovação tem acompanhado o espírito do tempo, de sorte que, hoje, inúmeros estudiosos de diversas áreas discutem um novo campo interdisciplinar para a pesquisa e sua respectiva prática, na atividade do design (CARDOSO, 2011; KISTMANN, 2014; MARENKO; BRASSETT, 2015).

A contemporaneidade vivencia o movimento pós-estruturalista [movimento referente à corrente que busca superar sua precedente estruturalista. Fortemente difundida pelo filósofo alemão F. Nietzsche e o francês M. Foucault, na década de 70 (MARENKO; BRASSETT, 2015)], que busca superar as estruturas, desconstruindo as "formas", as quais são estrategicamente construídas (todo tipo de estrutura, sistema), para pensar a reconstrução de um outro possível construto, ou seja, redesenhar o universo das possibilidades (DELEUZE; 
GUATTARI, 2008; MARENKO; BRASSETT, 2015). O produto passa pela desmaterialização e desenvolve valores intangíveis, como programas de softwares, estimulam sensações positivas, como a emoção, entre os artefatos, quer materiais, quer imateriais, como o aplicativo de celular ou objetos de marcas (ISSBERNER, 2010; COCCO, 2010; MARENKO; BRASSETT, 2015). Nesse contexto, a IoT se configura em uma das formas de interação desmaterializada.

\subsection{Conceito de Inovação no Design}

A maioria das definições do design atribui a tarefa de gerar formas materiais aos conceitos intelectuais. Atualmente, o campo do design abarca inúmeras áreas, para encontrar soluções eficientes (Inovação) entre tipos e relações, como é apresentado no diagrama da árvore do design, desenvolvido por David Walker, o qual realça as relações entre as áreas científicas, técnicas e artísticas (CARDOSO, 2011; BORJA MOZOTA, 2011; MARENKO; BRASSETT, 2015).

O conceito de inovação pressupõe criar algo novo que obtenha sucesso financeiro, seja no produto material, imaterial (serviço), seja no processo produtivo de desenvolvimento. Todos esses componentes são projetados com a finalidade de produzir inovações para melhor atender à demanda do mercado, integrando estratégias com foco no desempenho econômico e na criação de valor (COCCO, 2010; EL MARGHANI, 2013; ISSBERNER, 2010; KISTMANN, 2014; OECD, 2017).

O resultado competitivo pode ser adquirido, segundo Hamel e Prahalad (1989), a partir da identificação de tijolos faltantes no cenário futuro. Pode-se relacionar também com o território "puro" de Deleuze (2008), que trata do território ainda não estratificado ou sistematizado (dominado) pelo Estado [termo utilizado por Deleuze e Guattari, que indica força autoritária dada pelo homem, pelo seu interesse ou pela instituição (DELEUZE; GUATTARI, 2008)]. Com base nessa orientação, o design tem papel fundamental em estabelecer as competências direcionadas às empresas que constroem os seus produtos.

Gerar Inovação, no contemporâneo, pode ser contextualizado por Issberner (2010) e Cocco (2010), que tomam o capitalismo cognitivo como um novo paradigma do território de criação. Cocco traça o cenário desse capitalismo, inicialmente como uma camada estrutural, que comporta redes baseadas em tecnologias de informação e comunicação (TIC). De acordo com Cocco, o capitalismo cognitivo recorre às TIC para organizar e criar valor de produção dentro da própria rede de circulação (ISSBERNER, 2010; COCCO, 2010; ATZORI et al., 2010).

Como desdobramento, há outro aspecto do capitalismo cognitivo - o da rede enquanto meio de propagação de significados e valorização produtiva. A esse respeito, Cocco defende que a rede detém um papel semelhante ao da fábrica do capitalismo industrial (Sistema). A interação e a colaboração em rede são essenciais para o desenvolvimento do "novo", em meio à crise do valor, de maneira que se processam subterfúgios que gerem valor, por meio das dinâmicas cognitivas.

A Engenharia Kansei (KE), criada pelo professor japonês Mitsui Nagamachi, na década de 1970, surgiu nesse panorama de valorização da cognição, no Japão pós-guerras, saturado da industrialização fordiana (influência produtiva ocidental). Recorreu à apropriação da emoção, no processo da industrialização, reduzindo custos e otimizando resultados. Essa metodologia via Kansei (Kansei significa sentimento total) do usuário visa a compreender o ponto de vista do consumidor, para atender à demanda assertiva do mercado japonês, gerando inovação de produtos e processos (NAGAMACHI, 2011; SCHÜTTE, 2002). 
E assim, em paralelo, a internet das coisas (IoT) surge na expansão dessas contingências e será explicitada a seguir.

\subsection{Internet das coisas}

O conceito da Internet das coisas, traduzido da sigla em inglês IoT (Internet of Things), pode ser considerado como uma série de tecnologias disruptivas digitais, influenciando o cotidiano, tanto do indivíduo quanto dos negócios (ATZORI et al., 2010; KAMIENSKI et al., 2017). Instituições acadêmicas e empresas têm desenvolvido e adaptado tecnologias em seus processos de negociação, para obter resultados eficientes e inovadores, através do fluxo de informações. Essa prática tecnológica nada mais é do que o reflexo da emergência necessária da adaptação, dentre as mudanças que vêm ocorrendo no campo do design da atualidade (CARDOSO, 2011; MARENKO; BRASSETT, 2015).

Na prática da IoT, é essencial haver sinergia entre os vários campos do conhecimento, como a telecomunicação, a informática, a eletrônica e a ciência social. Com efeito, tem obtido resultados consideráveis quanto ao aumento de vinte e seis bilhões de objetos conectados por meio da IoT, até o ano de 2020. No viés econômico, há perspectiva de gerar em torno de um trilhão e novecentos mil dólares na produção de produtos e serviços que sustente $\mathrm{o}$ desenvolvimento do empreendimento (ATZORI et al., 2010; KAMIENSKI et al., 2017).

Existem várias perspectivas e exemplos aplicáveis da IoT. Entre os pontos fundamentais do empreendimento, está seguir a interoperatividade e a dinamicidade, contando com uma pesquisa ainda fragmentada. Para o sistema de gestão do conhecimento, uma implementação efetiva conta com três componentes: a infraestrutura física como hardware, componentes de software, extranet, intranet, entre outras conexões; tecnologia colaborativa, que permita reuniões, compartilhamento de dados, repositório de documentos; e a adoção da tecnologia de informação e comunicação (TIC), que pode ser integrada em diferentes tecnologias de colaboração, a qual possibilita a redução de custos entre as etapas produtivas e a atualização com respeito às informações, simultaneamente, entre os agentes da organização (ATZORI et al., 2010; DU et al., 2013; KAMIENSKI et al., 2017).

\subsubsection{Breve histórico da IoT}

Os objetos inteligentes possuem papel fundamental na evolução, tanto da pesquisa acadêmica quanto da mercadológica, porque esses objetos possuem capacidade de comunicação e processamento aliados a sensores, os quais transformam a utilidade desses objetos (MARENKO; BRASSETT, 2015; SANTOS et al., 2016).

$\mathrm{Na}$ IoT, a unidade básica de hardware apresenta ao menos uma das quatro seguintes características: a) unidade(s) de processamento; b) unidade(s) de memória; c) unidade(s) de comunicação e; d) unidade(s) de sensor(es) ou atuador(es). Os dispositivos com essas qualidades são denominados objetos inteligentes. Os objetos, ao estabelecerem comunicação com outros dispositivos, manifestam o conceito de estarem conectados (DU et al., 2013; SANTOS et al., 2016).

Ashton (2009 apud SANTOS et al., 2016), usou o termo Internet of Things pela primeira vez em seu trabalho na Procter \& Gamble, em 1999. Há cinco anos, a IoT era mais relacionada ao uso da tecnologia. Em 2005, o termo e a pesquisa começaram a tornar-se populares, quer pela academia, quer pela indústria. As mais populares foram os sensores sem fio. Essas conexões refletem nos avanços da automação residencial e industrial, bem como nas técnicas para explorar as diferentes limitações dos dispositivos, na escala e robustez da rede. Entre os anos 
de 2008 e 2010, o termo Internet das Coisas (IoT) ganhou maior espaço. Em 2012, foi previsto que a IoT levaria entre 5 e 10 anos para ser adotada pelo mercado, contudo, nos dias atuais, a prática é vivenciada, acima das expectativas, sobre a tecnologia (ATZORI et al., 2010; SANTOS et al., 2016).

Dentre essas "unidades" da IoT apresentadas, a inovação só é possível quando há um ambiente de confiabilidade, devendo-se considerar dois desafios: o da interoperatividade e o da agilidade de evolução. Para isso, é necessário ter em vista o estudo do sistema empregado e a correspondente arquitetura de funcionamento entre a relação homem-máquina, máquinamáquina, que gera requisitos empregados na resolução de novos construtos (SANTOS et al., 2016; KAMIENSKI et al., 2017).

O detalhamento mais técnico e aplicado não faz parte deste breve ensaio; aqui se procura refletir sobre o ambiente contemporâneo que gera inovação no design, por meio das novas metodologias. Num ponto de intersecção entre as duas tecnologias, existe um modelo interativo entre o homem e a casa inteligente, pela IoT, a qual configura um sistema que gera conforto numa casa, por meio da inteligência artificial, tendo como banco de dados a emoção.

\subsubsection{A emoção e sua respectiva mensuração, um caso específico de IoT}

A emoção e o design têm uma relação já desenvolvida desde o Design Emocional de Norman e Jordan, além de várias outras perspectivas profundas, como na psicanálise e na neurociência. Jordan (2000) define a emoção como reação fisiológica aos estímulos provocados do tálamo ao córtex vertebral, atingindo o hipotálamo, o qual determina as alterações neurovegetativas periféricas. Entre essas aspirações humanas, relacionam-se a criação, a emoção e a sinestesia, dentre os fenômenos. Ciente dessas considerações, o objetivo deste trabalho é tomar a emoção como um dos elementos que agregam valor ao construto contemporâneo (ZAJONC, 1980; JORDAN, 2000; NORMAN, 2004; EL MARGHANI et al., 2013).

O enfoque no desenvolvimento de pesquisas na emoção e na cognição de habilidades tem obtido crescentes resultados, na Ásia, Europa e, recentemente, nos EUA, baseados em teorias sobre o sistema interativo emocional entre o homem e a máquina no design (processo). No caso da IoT (inspirado na KE), apresentado por Du et al. (2013), o programa parte dos dados emocionais, como softwares aplicados à psicologia artificial, que provêm da inteligência artificial e se desenvolvem por caminhos transversais (DU et al., 2013; KAMIENSKI et al., 2017). Essa aplicação prática pode ser visualizada na Figura 1:

Figura 1. Plataforma do sistema com leitura emocional virtual. Fonte: Adaptado de Du et al., 2013.

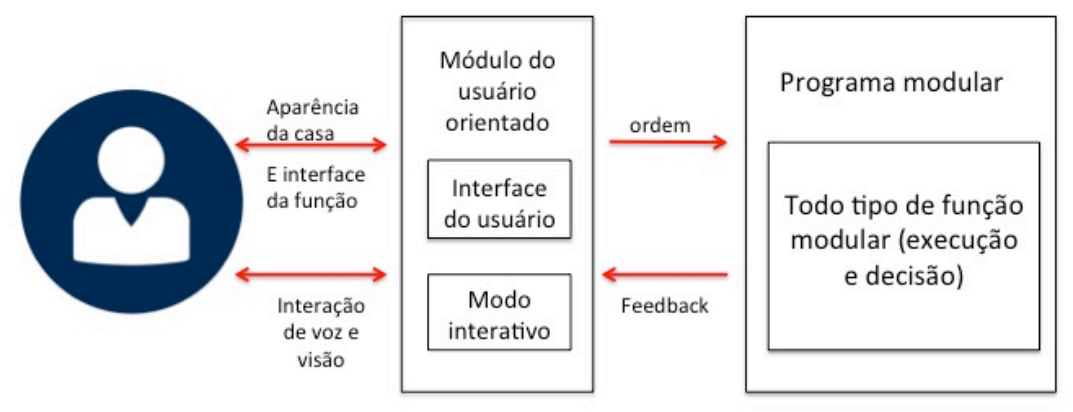


As expressões emocionais são divididas em duas categorias, conforme a teoria da psicologia: dimensional e categórica (ZAJONC, 1980). Por sua vez, Wilhelm Wundt foi um dos primeiros a configurar a teoria da emoção em três dimensões: prazer-desprazer; excitadoinibido; tenso-relaxado (ZAJONC, 1980).

Após definição desses estados opostos, de acordo com o postulado de Osgood (1965), mensura-se a graduação dessas emoções em até 16 unidades, ou seja, de -8 a 0 , de 0 a 8 , em escala de medida. Conforme a pontuação dos fatores dominantes, é possível calcular o estado emocional, positivamente e/ou negativamente, resultando em aspectos do espaço emocional. Com o resultado, o programa gera o estado emocional, personalidade, psicologia social, o que permite configurar preferências de mercado, satisfação e preferência de produto, entre outras aplicações (DU et al, 2013).

Um software de análise emocional, desenvolvido pelo Instituto de Informática e Tecnologia de Análise Léxica da China (ICTLAS - Institute of Computing Technology, Chinese Lexical), divide os estados emocionais em nove categorias: calmo, feliz, excitado, triste, choroso, irado, frustrado, surpreso e medroso, após definição da segmentação de categoria da "palavra emocional", seja verbo, seja adjetivo, advérbio, interjeição e/ou conjunção. A partir dos dados de entrada dessas palavras emocionais, pode-se construir um novo modelo de satisfação com as seguintes etapas:

- O texto é inserido pelo computador;

- O texto é segmentado, e as palavras são extraídas dentre 7 categorias;

- De acordo com as bases de dados emocionais, as palavras das 7 categorias são classificadas em 9 tipos de estados emocionais;

- Calcula-se entre as palavras que representam o estado positivo e negativo das emoções;

- Calcula-se a intensidade das palavras emocionais. Procedimentos e dados cruzados complexos que apenas o algoritmo (lógica fuzzy, rede neural etc.) configura no resultado otimizado, considerando todas as variáveis inseridas no processo (ISHIHARA, 1995);

- A probabilística representativa da proporção emocional do resultado apresenta uma sentença visualizável.

\subsection{Engenharia Kansei}

Imersa nesse contexto de mercado, a Engenharia Kansei (KE) surgiu na década de 1970, criada pelo professor e engenheiro de produção japonês Mitsui Nagamachi, da Universidade de Hiroshima, Japão. A KE trata de uma efetiva técnica que traduz as emoções e os desejos do usuário/consumidor (U/C) em novos elementos de produto. $\mathrm{O} \mathrm{U} / \mathrm{C}$ pode experimentar sensações de maior prazer no uso do produto/serviço implementado pela KE.

Nagamachi (2011) afirma que a KE pode ser implementada para a produção de produtos (todas as camadas do design, da roupa à arquitetura), tendo em vista as impressões sensoriais fisiológicas tratáveis no processo, o qual reflete emoções nas soluções específicas em parâmetros de design.

A seguir, as três etapas de concepção do construto esperado (SHÜTTE, 2002):

- Aplica-se o diferencial semântico (OSGOOD, 1965) como técnica que entenda os sentimentos do $\mathrm{U} / \mathrm{C}$; 
- O levantamento é conduzido para encontrar relações entre as palavras Kansei e os atributos/elementos de design

- Com o auxílio do computador, pode-se desenvolver o processo sistemático da KE, a qual transforma as palavras Kansei em elementos de design.

A ideia básica de descrição do conceito do produto provém de duas perspectivas diferentes: a descrição semântica e a descrição das propriedades do produto. Esses dois vetores espaciais de descrição indicam quais das propriedades de produto evocam o impacto de diferencial semântico (SCHÜTTE, 2002; EL MARGHANI et al., 2013). O primeiro passo é validar as propriedades que sintetizam e atualizam o sistema, para ativá-lo novamente. Quando os resultados dessa interação processual são aceitos, o modelo preditivo constrói a semântica da relação com o espaço da propriedade aplicada, conforme a Figura 2.

Figura 2. Modelo básico de procedimento da Engenharia Kansei. Fonte: Adaptado de Schütte, 2002.

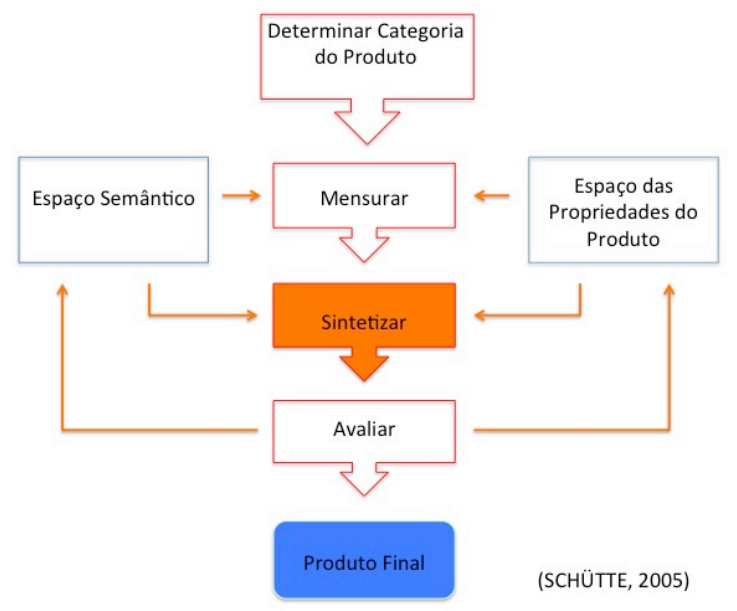

A partir desse modelo conceitual, existem seis tipos de KE, de acordo com a complexidade do construto a ser desenvolvido. Por exemplo, o procedimento da fabricação de uma caneta difere, expansivamente, das etapas da construção de uma aeronave, mesmo com as etapas equivalentes do desenvolvimento de produto. Para a compreensão dos tipos KE, serão citados alguns exemplos de produtos, respectivamente com o tipo em que se adequam na produção.

\subsubsection{Tipos de Engenharia Kansei}

Em 1995, apenas três tipos de KE suportavam a produção industrial, desde a década de 1970: a do tipo classificatório, a de sistema computacional e a de modelo. Desde meados da década de 90, outros três modelos foram incorporados à metodologia, conforme o surgimento de novas necessidades, a que as tecnologias se adequam e suprem as soluções. Os tipos aplicados são: Classificação, Sistemática, Híbrido, Matemático, Virtual e Colaborativo. O tipo Colaborativo será mais bem enfatizado, para posterior relação com o caso específico da IoT mencionado anteriormente.

O modelo básico de KE, segundo Nagamachi (2011), é a base da metodologia pela qual uma categoria de produto Kansei divide a estrutura, para identificar o detalhe específico do design. Através das palavras Kansei, o U/C é encorajado a expressar seu sentimento e estado 
emocional. Esse método é denominado diferencial semântico (SHÜTTE, 2002; EL MARGHANI et al., 2013).

Segundo se observou, o sexto tipo é a KE colaborativa, exclusivamente desenvolvido no ambiente digital, pela internet, pois necessita de softwares específicos (tecnologia mencionada no ambiente da IoT) que proporcionem o acesso de vários designers e colaboradores, no desenvolvimento processual do produto. Com o uso da internet, é possível construir uma estrutura de grupo, um sistema com base de dados Kansei. Esse tipo de KE colaborativa será o método de análise comparativa à atividade da IoT.

\section{MÉTODO - ANÁLISE COMPARATIVA ENTRE A KE e a IoT}

O objetivo deste tópico é realizar aproximações sintéticas e destacar as diferenças desses dois estudos atuais. Como visto anteriormente, tanto a KE quanto a IoT trabalham no ambiente virtual e colaborativo, incorporando pessoas, ferramentas, tecnologias, dispositivos e equipamentos. Busca-se apresentar, no campo em comum às duas pesquisas, a formulação da inovação do design contemporâneo através da internet e da emoção, de que resultam novos construtos.

De acordo com o método de pesquisa no design (GIL, 1989; DRESCH, 2015; BRYMAN, 2016), é possível realizar uma análise de conteúdo comparativa (compilada) entre dois modelos teóricos por relação conceitual, natureza, codificação e indicação de variabilidade.

A IoT é um objeto tecnológico criado pelo homem, com o objetivo de suprir as necessidades estendidas, no mundo contemporâneo. A sociedade incorporou esse comportamento imperativo, para dinamizar o processo da busca de qualidade de vida e solução, por todo o ambiente do design contemporâneo, dentre produtos tangíveis (hardware) e intangíveis (software).

A KE do tipo colaborativo é uma metodologia de desenvolvimento de produto específico que enseja a interação entre o homem e a máquina, a fim de gerar construtos novos, considerando o banco de dados da emoção.

Segue a tabela comparativa entre o estudo da IoT (o caso referido) e o método KE colaborativo, responsáveis em gerar inovação no campo do design, com a sistematização emocional no ambiente virtual:

Tabela 1. Síntese comparativa entre a IoT do sistema interativo emocional e a KE colaborativa. Fonte: Elaborada pelas autoras baseada em Gil (1989); Dresch (2015); Bryman (2016).

\begin{tabular}{|c|c|c|c|c|}
\hline Tema & Conceito & Natureza & $\begin{array}{c}\text { Codificação/ } \\
\text { Interpretação }\end{array}$ & $\begin{array}{c}\text { Indicação de } \\
\text { variabilidade }\end{array}$ \\
\hline $\begin{array}{c}\text { IoT sistema } \\
\text { interativo }\end{array}$ & $\begin{array}{c}\text { Simétrico, } \\
\text { Assimétrico e } \\
\text { Recíproco }\end{array}$ & $\begin{array}{c}\text { Objeto/ } \\
\text { Tecnologia }\end{array}$ & $\begin{array}{c}\text { Abrange todo o } \\
\text { ambiente } \\
\text { Processual }\end{array}$ & $\begin{array}{c}\text { tecnológico do } \\
\text { Design }\end{array}$ \\
\hline KE colaborativo & Simétrico & Metodologia & Processual & $\begin{array}{c}\text { Metodologia } \\
\text { inserida no } \\
\text { ambiente da IoT }\end{array}$ \\
\hline
\end{tabular}


Conforme os métodos de pesquisa (GIL, 1989; DRESCH, 2015; BRYMAN, 2016), pode-se visualizar o resumo elaborado da configuração comparativa da Tabela 1. A relação conceitual da IoT, no sistema interativo, é considerada narrativa simétrica à ideia de sistematização da emoção com a KE colaborativa, sendo empregada a interação e a interoperatividade entre o homem e a máquina, e a máquina (programa software) que produz design inovador. A assimetria conceitual situa quanto à dimensão proporcional, pois a IoT abrange todo um ambiente tecnológico de desenvolvimento da inovação entre todas as camadas do design, enquanto a KE colaborativa consiste em uma metodologia que desenvolve inovação, num determinado produto, com a interação homem-máquina. Ademais, há reciprocidade qualitativa entre as propriedades teóricas, porque as duas tecnologias estão gerando construtos ótimos e ideais, por meio da tecnologia das máquinas (mensuração matemática, software), aferindo validade, qualidade e precisão na inovação.

\section{CONSIDERAÇÕES FINAIS}

Este trabalho partiu do questionamento se seria possível construir inovação em rede, considerando a IoT, desenvolvidas por meio da interoperabilidade, e a sistematização da emoção pela metodologia KE colaborativa. $\mathrm{O}$ estudo demonstra que no ambiente em que se gera inovação, no contexto do design contemporâneo, tem o intuito de levantar reflexão acerca de elementos tais como a colaboração, emoção e a rede, antes impensados na industrialização do mundo real de V. Papanek (CARDOSO, 2011). A emoção e o estado psicológico podem ser tratados através de procedimentos aritméticos geradores de novas formas e construtos satisfatórios na excelência em design, sem fronteiras geográficas nem étnicas.

Existem limitações técnicas, no funcionamento corrente, como flutuações dos sinais e suportes no ambiente virtual, bem como entre as etapas processuais da produção, contudo, é possível criar, reconstruir, com esses agenciamentos entre as máquinas produtoras de inovação no design contemporâneo. Eis o universo "inexistente" (termo utilizado por HAMEL \& PRAHALAD, 1989), que faz surgir conexões entre pensamento imanente e prático, as quais sobrepõem questões conceituais: afinal, a atividade do design é a prática processual de criar mundos.

\section{REFERÊNCIAS}

ATZORI, L. et al. The Internet of Things: A survey. Computer Networks, v54, n.15, p. 2787$2805,2010$.

BORJA DE MOZOTA, B. Gestão do design: usando o design para construir valor de marca e inovação corporativa. Porto Alegre: Bookman, 2011. 337 p.

BRYMAN, A. Social Research Methods. 5th ed. New York: Oxford University Press, 2016. $747 \mathrm{p}$.

CARDOSO, R. Design para um mundo complexo. São Paulo: Cosac \& Naify, 2011. 263 p.

COCCO, G. Indicadores de Inovação e capitalismo cognitivo. In: Bases conceituais em pesquisa, desenvolvimento e inovação: implicações para política no Brasil - Brasília: Centro de Gestão e Estudos Estratégicos, p. 33-67, 2010.

DELEUZE, G.; GUATTARI, F. Mil Platôs. Capitalismo e Esquizofrenia. 4. reimpr. v. 5. São Paulo: 34, 2008. 235 p. 
DRESCH, A. et al. Design Science Research. Método de pesquisa para avanço da ciência e tecnologia. Porto Alegre: Bookman, 2015. 181 p.

DU, K. et al. Human interactive machine system on smart home of IoT. The Journal of China Universities of Posts and Telecommunications. Elsevier, v20, n. 1, p. 96-99, 2013.

EL MARGHANI, V. et al. Emotional Engineering. In: FUKUDA, Shuichi (Org.). Kansei Engineering: Methodology to the Project Oriented for the Customers, v. 2. London: Springer, 2013. p. 107-148.

GIL, A. C. Métodos e técnicas de pesquisa social. 2. ed. São Paulo: Atlas, 1989. 105 p.

ISHIHARA, H. et al. An automatic builder for a Kansei Engineering expert system using selforganizing neural networks. Int J Ind Ergon, v111, n. 15. p. 13-24, 1995.

ISSBERNER, R.L. Em direção a nova abordagem da inovação: Coordenada para o debate. In: Bases conceituais em pesquisa, desenvolvimento e inovação. Implicações para políticas no Brasil - Brasília: Centro de Gestão e Estudos Estratégicos. p. 11-31. 2010.

HAMEL, G.; PRAHALAD, G. K. Strategic intent. Harvard Business Review, 1989. 101 p.

JORDAN, P. W. Designing Pleasurable Products: An introduction to the new human factors. London; New York: Taylor \& Francis, 2000. 224 p.

KAMIENSKI, C. et al. Application development for the Internet of Things: A context-aware mixed criticality systems development platform. Computer Communications, v104, n.17, p. 1$16,2017$.

KISTMANN, V.B. Interdisciplinaridade: questões quanto à pesquisa e à inovação em design. Estudos em Design (online). Rio de Janeiro: v22. n.3, p. 81-99, 2014.

NAGAMACHI, M. Kansei/Affetive Engineering and History of Kansei Engineering in the World. In: NAGAMACHI, M. (Ed.). Kansei/Affective Engineering. Boca Raton: Taylor \& Francis, 2011. 320 p.

NORMAN, D. A. Emotional design: why we love (or hate) every day things. New York: Basic Books, 2004.

OECD. Science and Innovation. Brazil, 2012. 117 p.

OSGOOD C. E. The nature and measurement of meaning. In: OSGOOD, C. E.; SNIDER, J. G. (Ed.). Semantic Differential Technic - A source book. Chicago: Aldine, p. 3-41. 1965.

SANTOS, B. P. et al. Internet das coisas: da teoria à prática. Departamento de Ciência da Computação - Instituto de Ciências Exatas Universidade Federal de Minas Gerais (UFMG) Belo Horizonte: Editora UFMG, p.1-52, 2016.

SHÜTTE, S. Designing Feelings into Products: Integrating Kansei Engineering Methodology in Product Development. 2002. 115f. Tese (PhD) - Course of Integrating Kansei Engineering Methodology in Product Development, Department of Mechanical Engineering, Linköpings Universitet, Linköping, 2002.

ZAJONC, R. B. Feeling and Thinking: Preferences Need No Inferences. American Psychologist. v35, n.2, p.151-175, 1980. 